\title{
مجتمع الباحثين
}

\section{رسائل الماجستير التى تم تسجيلها في قسم الصحافة:-}

متابعة : أ. ياسمين أبو العلا"

- تأثير أجندة الصحف المحلية ومواقعها الإلكترونية على اتجاهات الجمهور فى : صعيد مصر : در اسة تحليلية مقارنة للصحف و القائم بالاتصال و الجمهور فى الصى الفترة من 2013 إلى 2014.

- علاقة السياسة الخارجية التركية تجاه مصر بصورة تركيا فى خطاب الصحافة المصرية بعد ثورتى 25 يناير و30 يونيو: دراسة تحليلية خلال الفترة من

$$
\text { يناير } 2011 \text { إلى ديسمبر } 2014 .
$$

- العو امل المؤثرة على قارئية صفحات الحوادث بالصحف المصرية: دراسة للجمهور و القائم بالاتصال. - اعتماد رجال الأعمال على وسائل الاتصال بتشكيل اتجاهاتهم نحو الاتثمار فى

$$
\text { الصعيد: در اسة مبدانية. }
$$

- أطر تقديم العلاقات المصرية الفلسطينية فى الصحافة المصرية وعلاقاتها باتجاهات الجمهور نحو القضية الفلسطينية.

- أطر تقديم مؤسسة القضاء فى الصحافة وعلاقتها بالصورة الذهنية للقضاة لدى الجمهور . - العلاقة بين مصداقية شبكات التو اصل الاجتماعى و الصفحات الاخبارية الرسمية و اعتماد الثباب عليها كمصدر للمعلومات: در اسة تحليلية ميدانية. - آليات إدارة الأزمات الاقتصادية المحلية فى الصحف المصرية وعلاقاتها بتشكيل اتجاهات النخبة نحو الأزمتان. رسائل الاكتور اه التى تم تسجيلها فيى قسم الصحافة:- العوامل المؤثرة على مضمون الصفحة الأولى فى الصحف المصرية وشكلها خلال الفترة من 1996 - 2011.

"المدرس المساعد بقسم الصحافة - بكلية الإعلام - جامعة القاهرة. 
- دور تكنولوجيا المعلومات الصحفية فى تطوير الخدمة الإخبارية على المواقع الإلكترونية وشبكات التو اصل الاجتماعى: در اسة تحليلية ميدانية على الصحافة

$$
\text { الإلكترونية السورية ومستخدميها. }
$$

- أطر المعالجة الصحفية لشئون المؤسستين العسكرية والأمنية المصريتين: دراسة تحليلية على عينة من الصحف العربية والأجنبية فى الفترة من 2014/2011. - المعايير الأخلاقية و القانونية لأساليب تعامل الصحفيين مع مصادر المعلومات و علاقنها بالسياسات التحريرية لعينة من الصحف المصرية والأمريكية و البريطانية نحو نموذج مهنى لتطوير أداء القائم بالاتصال.

- أطر التغطية الخبرية للأحداث الجارية فى الصحافة المصرية: دراسة لآليات التحيز فى بو ابات الصحف الإلكترونية.

\section{أحلث الكتب الإنجليزية التى تم إضافتها لمكتبة كلبة الإعلام:-}

- Heather Bowe, Kylie Martin, Howard Manns, "Communication Across Cultures: Mutual Understanding in a global World", $2^{\text {nd }}$ edition, Cambridge University, 2014

- Antra Arora, "Communication Media and Internet", India: Random Publications, 2015

- Brian L. Ott and Robert L. Mach, "Critical Media Studies: An Introduction", $2^{\text {nd }}$ edition, Wiley Blackwell, 2014.

- Steve Schifferes and Richard Roberts (Editors), "The Media and Financial Crisis: Comparative and Historical Perspective", New York: Routledge,2015

- Stephen M.Croucher and Daniel Cronn- Mills, "Understanding Communication Research Methods: A Theoratical and Practical approach", New York: Routledge, 2015 
مجتمع الباحثين

$$
\text { كتب عريبة:- }
$$

- محمود قلندر ، "وسائل الاتصال والمجتمع"، ط1، مكتبة الفلاح، 2015 - أيمن عبد الهادى، "وسائل الاتصال"، ابداع للنشر والتوزيع، 2015 - - نجلاء عبدالفتاح طه، دور الإعلام فى حل القضايا المعاصرة، مكتبة العربى، ولئ 2015

- أثرف صالح، تصميم المطبوعات الإعلامية، دار النهضة العربية، 2015

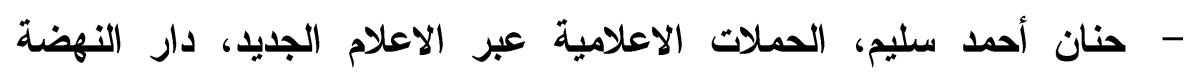
العربية، 2015 - (20) 